\title{
Existence and Uniqueness of Mild Solutions to Some Neutral Stochastic Partial Functional Integrodifferential Equations with Non-Lipschitz Coefficients
}

\author{
Mamadou Abdoul Diop, ${ }^{1}$ Khalil Ezzinbi, $^{2}$ and Modou Lo ${ }^{1}$ \\ ${ }^{1}$ Département de Mathématiques, UFR SAT, Université Gaston Berger de Saint-Louis, \\ P.O. Box 234, Saint-Louis, Senegal \\ ${ }^{2}$ Département de Mathématiques, Faculté des Sciences Semlalia, Université Cadi Ayyad, \\ P.O. Box 2390 Marrakech, Morocco \\ Correspondence should be addressed to \\ Mamadou Abdoul Diop, mamadou-abdoul.diop@ugb.edu.sn
}

Received 9 November 2011; Revised 17 January 2012; Accepted 25 January 2012

Academic Editor: Charles E. Chidume

Copyright (C) 2012 Mamadou Abdoul Diop et al. This is an open access article distributed under the Creative Commons Attribution License, which permits unrestricted use, distribution, and reproduction in any medium, provided the original work is properly cited.

This paper presents the result on existence, uniqueness of mild solutions to neutral stochastic partial functional integrodifferential equations under the Carathéodory-type conditions on the coefficients. The results are obtained by using the method of successive approximation. An example is provided to illustrate the results of this work.

\section{Introduction}

In this paper, our objective is to study the existence of mild solution for the following neutral stochastic partial functional integrodifferential equation in a real separable Hilbert space:

$$
\begin{aligned}
d\left[u(t)-H\left(t, u_{t}\right)\right]= & A\left[u(t)-H\left(t, u_{t}\right)\right] d t+\left[\int_{0}^{t} B(t-s)\left[u(s)-H\left(s, u_{s}\right)\right] d s+F\left(t, u_{t}\right)\right] d t \\
& +G\left(t, u_{t}\right) d w(t), \quad \text { for } t \in[0, T], \\
u_{0}(\cdot)= & \varphi \in C_{q_{0}}^{b}([-r, 0] ; \mathbb{H}), \quad r>0,
\end{aligned}
$$

where $u_{t}(\theta)=u(t+\theta)$ for $\theta \in[-r, 0]$. The mappings $H: \mathbb{R}_{+} \times C_{r} \rightarrow \mathbb{H}, F: \mathbb{R}_{+} \times C_{r} \rightarrow \mathbb{H}$, and $G: \mathbb{R}_{+} \times C_{r} \rightarrow \mathcal{L}(\mathbb{K}, \mathbb{H})$ are Borel measurable. 
Neutral stochastic partial functional differential equations have atracted great interest due to their applications in characterizing many problems in physics, biology, mechanics and so on.

Qualitative properties such as existence, uniqueness, and stability for various stochastic differential and integrodifferential systems have been extensively studied by many researchers; see, for instance, [1-6] and the references therein. The problem of the existence and uniqueness of solution for neutral stochastic partial functional differential equations in the case where the coefficients do not satisfy the global Lipschitz condition was investigated by Taniguchi [7], Turo [8], Cao et al. [9], and recently by Jiang and Shen [10].

Stimulated by the above works, we consider the existence and uniqueness of mild solutions to (1.1) under some carathéodory-type conditions to the Hilbert space with the Lipschitz condition in [11] and the non-Lipschitz condition in [12] being regarded as special cases.

Our main results concerning (1.1) rely essentially on techniques using strongly continuous family of operators $\{R(t), t \geq 0\}$, defined on the Hilbert space $\mathbb{H}$ and called the resolvent (precise definition will be given below).

The contents of the paper are as follows. In Section 2, we summarize several important working tools on the Wiener process and deterministic integrodifferential equations that will be used to develop our results. In Section 3, we study the existence of mild solutions for the neutral system (1.1) using the theory of resolvent operators and by means of successive approximation (the Picard iteration). In Section 4, we provide an example to illustrate our main approach.

\section{Wiener Process and Deterministic Integrodifferential Equations}

\subsection{Wiener Process}

Throughout this paper, let $\left(\Omega, \mathcal{F},\left\{\mathcal{F}_{t}\right\}_{t \geq 0}, \mathbb{P}\right)$ be a complete probability space with a normal filtration $\left\{\mathcal{F}_{t}\right\}_{t \geq 0}$ satisfying the usual conditions (i.e., it is increasing and right-continuous while $\mathcal{F}_{0}$ contains all $\mathbb{P}$-null sets). Moreover, let $\mathbb{H}$ and $\mathbb{K}$ be two real separable Hilbert spaces; we denote by $\langle\cdot, \cdot\rangle_{\mathbb{H}},\langle\cdot, \cdot\rangle_{\mathbb{K}}$ their inner products and by $\|\cdot\|_{\mathbb{H}},\|\cdot\|_{\mathbb{K}}$ their vectors norms, respectively. We denote by $\mathcal{L}(\mathbb{H}, \mathbb{K})$ the space of all bounded linear operator from $\mathbb{H}$ into $\mathbb{K}$, equipped with the usual operator norm $\|\cdot\|$. In this paper, we always use the same symbol $\|\cdot\|$ to denote norms of operators regardless of the spaces potentially involved when no confusion possibly arises. Let $r>0$ and $C_{r}=C([-r, 0] ; \mathbb{H})$ denote the family of all continuous $\mathbb{H}$-valued functions $\xi$ from $[-r, 0]$ to $\mathbb{H}$ with norm $\|\xi\|_{C}=\sup _{t \in[-r, 0]}\|\xi(t)\|_{\mathbb{H}}$. Let $C_{q_{0}}^{b}([-r, 0] ; \mathbb{H})$ be the family of all almost surely bounded, $\mathcal{F}_{0}$-measurable, $C([-r, 0] ; \mathbb{H})$-valued random variables.

Let $\{w(t): t \geq 0\}$ denote a $\mathbb{K}$-valued Wiener process defined on the probability space $\left(\Omega, \mathcal{F},\left\{\mathcal{F}_{t}\right\}_{t \geq 0}, \mathbb{P}\right)$ with covariance operator $Q$; that is, $E\langle w(t), x\rangle_{\mathbb{K}}\langle w(s), y\rangle_{\mathbb{K}}=(t \wedge s)\langle Q x, y\rangle_{\mathbb{K}}$, for all $x, y \in \mathbb{K}$, where $Q$ is a positive, self-adjoint, trace class operator on $\mathbb{K}$. In particular, we denote $w(t)$ a $\mathbb{K}$-valued $Q$-Wiener process with respect to $\left\{\mathscr{F}_{t}\right\}_{t \geq 0}$. To define stochastic integrals with respect to the $Q$-Wiener process $w(t)$, we introduce the subspace $\mathbb{K}_{0}=$ $Q^{1 / 2} \mathbb{K}$ of $\mathbb{K}$ endowed with the inner product $\langle u, v\rangle_{\mathbb{K}_{0}}=\left\langle Q^{-1 / 2} u, Q^{-1 / 2} v\right\rangle_{\mathbb{K}}$ as a Hilbert space. We assume that there exists a complete orthonormal system $\left\{e_{i}\right\}$ in $\mathbb{K}$, a bounded sequence of nonnegative real numbers $\lambda_{i}$ such that $Q e_{i}=\lambda_{i} e_{i}, i=1,2, \ldots$, and a sequence $\left\{\beta_{i}(t)\right\}_{i>1}$ of independent standard Brownian motions such that

$$
w(t)=\sum_{i=1}^{+\infty} \sqrt{\lambda_{i}} \beta_{i}(t) e_{i}, \quad t \geq 0
$$


and $\mathcal{F}_{t}=\mathcal{F}_{t}^{w}$, where $\mathcal{F}_{t}^{w}$ is the $\sigma$-algebra generated by $\{w(s): 0 \leq s \leq t\}$. Let $\mathcal{L}_{2}^{0}=\mathcal{L}_{2}\left(\mathbb{K}_{0}, \mathbb{H}\right)$ be the space of all Hilbert-Schmidt operators from $\mathbb{K}_{0}$ to $\mathbb{H}$. It turns out to be a separable Hilbert space equipped with the norm $\|v\|_{\mathcal{L}_{2}^{0}}=\operatorname{tr}\left(\left(v Q^{1 / 2}\right)\left(v Q^{1 / 2}\right)^{*}\right)$ for any $v \in \mathcal{L}_{2}^{0}$. Obviously, for any bounded operator $v \in \mathcal{L}_{2}^{0}$, this norm reduces to $\|v\|_{\mathcal{L}_{2}^{0}}^{2}=\operatorname{tr}\left(v Q v^{*}\right)$.

\subsection{Partial Integrodifferential Equations}

In this section, we recall some fundamental results needed to establish our results. Regarding the theory of resolvent operators, we refer the reader to $[13,14]$. Throughout the paper, $X$ is a Banach space $A$ and $B(t)$ are closed linear operators on $X . Y$ represents the Banach space $D(A)$ equipped with the graph norm defined by

$$
|y|_{Y}:=|A y|+|y| \text { for } y \in Y \text {. }
$$

The notations $C([0,+\infty) ; Y), B(Y, X)$ stand for the space of all continuous functions from $[0,+\infty)$ into $Y$, the set of all bounded linear operators from $Y$ into $X$, respectively. We consider the following Cauchy problem:

$$
\begin{gathered}
v^{\prime}(t)=A v(t)+\int_{0}^{t} B(t-s) v(s) d s, \text { for } t \geq 0, \\
v(0)=v_{0} \in X .
\end{gathered}
$$

Definition 2.1 (see [13]). A resolvent operator for (2.3) is a bounded linear operator-valued function $R(t) \in \mathcal{L}(X)$ for $t \geq 0$, having the following properties.

(i) $R(0)=I$ and $|R(t)| \leq N e^{\beta t}$ for some constants $N$ and $\beta$.

(ii) For each $x \in X, R(t) x$ is strongly continuous for $t \geq 0$.

(iii) $R(t) \in \mathcal{L}(Y)$ for $t \geq 0$. For $x \in Y, R(\cdot) x \in C^{1}([0,+\infty) ; X) \cap C([0,+\infty) ; Y)$ and

$$
\begin{aligned}
R^{\prime}(t) x & =A R(t) x+\int_{0}^{t} B(t-s) R(s) x d s \\
& =R(t) A x+\int_{0}^{t} R(t-s) B(s) x d s, \quad \text { for } t \geq 0 .
\end{aligned}
$$

In what follows we suppose the following assumptions.

(H1) A is the infinitesimal generator of a strongly continuous semigroup on $X$.

(H2) For all $t \geq 0, B(t)$ is closed linear operator from $D(A)$ to $X$ and $B(t) \in B(Y, X)$. For any $y \in Y$, the map $t \rightarrow B(t) y$ is bounded and differentiable, and the derivative $t \rightarrow B^{\prime}(t) y$ is bounded uniformly continuous on $\mathbb{R}^{+}$.

The resolvent operator plays an important role to study the existence of solutions and to give a variation of constants formula for nonlinear systems. We need to know when the linear system 2.1 has a resolvent operator. For more details on resolvent operators, we refer 
to $[13,14]$. The following theorem gives a satisfactory answer to this problem, and it will be used in this work to develop our main results.

Theorem 2.2 (see [13]). Assume that (H1)-(H2) hold. Then there exists a unique resolvent operator of the Cauchy problem (2.3).

In the following, we give some results for the existence of solutions for the following integrodifferential equation:

$$
\begin{gathered}
v^{\prime}(t)=A v(t)+\int_{0}^{t} B(t-s) v(s) d s+q(t), \text { for } t \geq 0 \\
v(0)=v_{0} \in X
\end{gathered}
$$

where $q:[0,+\infty[\rightarrow X$ is a continuous function.

Definition 2.3 (see [13]). A continuous function $v:[0,+\infty) \rightarrow X$ is said to be a strict solution of (2.5) if

(i) $v \in C^{1}([0,+\infty) ; X) \cap C([0,+\infty) ; Y)$,

(ii) $v$ satisfies (2.5), for $t \geq 0$.

Remark 2.4. From this definition, we deduce that $v(t) \in D(A)$, the function $B(t-s) v(s)$ is integrable, for all $t \geq 0$, and $s \in[0, \mathrm{t}]$.

Theorem 2.5 (see [13]). Assume that (H1)-(H2) hold. If $v$ is a strict solution of (2.5), then

$$
v(t)=R(t) v_{0}+\int_{0}^{t} R(t-s) q(s) d s, \quad \text { for } t \geq 0
$$

Accordingly, we make the following definition.

Definition 2.6 (see [13]). For $v_{0} \in X$, a function $v:[0,+\infty) \rightarrow X$ is called a mild solution of (2.5) if $v$ satisfies (2.6).

The next theorem provides sufficient conditions for the regularity of solutions of (2.5).

Theorem 2.7 (see [13]). Let $q \in C^{1}([0,+\infty) ; X)$ and $v$ be defined by (2.6). If $v_{0} \in D(A)$, then $v$ is a strict solution of (2.5).

\section{Existence of Mild Solutions of (1.1)}

Definition 3.1. A process $\{u(t), t \in[0, T]\}, 0 \leq T<+\infty$, is called a mild solution of (1.1) if

(i) $u(t)$ is $\mathcal{F}_{t}$-adapted, $t \geq 0$ with $\int_{0}^{T}\|u\|_{\mathbb{H}}^{2} d t<+\infty$ a.s.; 
(ii) $u(t) \in \mathbb{H}$ has continuous paths on $t \in[0, T]$ a.s., and, for each $t \in[0, T], u(t)$ satisfies the integral equation

$$
\begin{aligned}
u(t)-H\left(t, u_{t}\right)= & R(t)[\varphi(0)-H(0, \varphi)]+\int_{0}^{t} R(t-s) F\left(s, u_{s}\right) d s \\
& +\int_{0}^{t} R(t-s) G\left(s, u_{s}\right) d w(s),
\end{aligned}
$$

for any $u_{0}(\cdot)=\varphi \in C_{\Psi_{0}}^{b}([-r, 0] ; \mathbb{H})$.

In the rest of this paper we replace $X$ by $\mathbb{H}$ in $(\mathrm{H} 1)$ and (H2).

To guarantee the existence and uniqueness of a mild solution to (1.1), the following much weaker conditions, instead of the non-Lipschitz condition, are used.

(H3) (a) There exists a function $L(t, y): \mathbb{R}_{+} \times \mathbb{R}_{+} \rightarrow \mathbb{R}_{+}$such that $L(t, y)$ is locally integrable in $t \geq 0$ and is continuous monotone nondecreasing and concave in $y$ for any fixed $t \in[0, T]$. Moreover, for any fixed $t \in[0, T]$ and $\zeta \in \mathbb{H}$, the following inequality is satisfied:

$$
\|F(t, \zeta)\|_{\mathbb{H}}^{2}+\|G(t, \zeta)\|_{\mathcal{L}_{2}^{0}}^{2} \leq L\left(t,\|\zeta\|_{C}^{2}\right), \quad t \in[0, T]
$$

(b) For any constant $K^{*}>0$, the differential equation

$$
\frac{d x}{d t}=K^{*} L(t, x), \quad t \in[0, T]
$$

has a global solution for any initial value $x_{0}$.

(H4) (a) There exists a function $\lambda(t, y): \mathbb{R}_{+} \times \mathbb{R}_{+} \rightarrow \mathbb{R}_{+}$such that $\lambda(t, y)$ is locally integrable in $t \geq 0$ for any fixed $y \geq 0$ and is continuous monotone nondecreasing and concave in $y$ for any fixed $t \in[0, T] . G(t, 0)=0$ for any fixed $t \in[0, T]$. Moreover, for any fixed $t \in[0, T]$ and $\zeta, \eta \in \mathbb{H}$, the following inequality is satisfied:

$\|F(t, \zeta)-F(t, \eta)\|_{\mathbb{H}}^{2}+\|G(t, \zeta)-G(t, \eta)\|_{\mathcal{L}_{2}^{0}}^{2} \leq \lambda\left(t,\|\zeta-\eta\|_{C}^{2}\right), \quad t \in[0, T]$.

(b) For any constant $\bar{K}>0$, if a nonnegative function $z(t)$ satisfies that

$$
z(t) \leq \bar{K} \int_{0}^{t} \lambda(s, z(s)) d s, \quad t \in[0, T]
$$

then $z(t)=0$ holds for any $t \in[0, T]$.

(H5) The mapping $H(t, x)$ satisfies that there exists a positive $K$ such that, for any $\zeta, \eta \in$ $\mathbb{H}$ and $t \geq 0$,

$$
\|H(t, \zeta)-H(t, \eta)\|_{\mathbb{H}} \leq K\|\zeta-\eta\|_{C}
$$


Remark 3.2. Let $\lambda(t, u)=\beta(t) \bar{\lambda}(u), t \in[0, T]$, where $\beta(t) \geq 0$ is locally integrable and $\bar{\lambda}(u)$ is a concave nondecreasing function from $\mathbb{R}_{+}$to $\mathbb{R}_{+}$such that $\bar{\lambda}(0)=0, \bar{\lambda}(u)>0$ for $u>$ 0 and $\int_{0^{+}}(1 / \bar{\lambda}(u)) d u=\infty$. Then, by the comparison theorem of differential equations we know that assumption (H4-(b)) holds.

Now let us give some concrete examples of the function $\bar{\lambda}(\cdot)$. Let $\xi>0$ and let $\delta \in$ $(0,1)$ be sufficient small. Define

$$
\begin{gathered}
\overline{\mathcal{\lambda}}_{1}(u)=\xi u, \quad u \geq 0, \\
\overline{\mathcal{\lambda}}_{2}(u)= \begin{cases}u \log \left(u^{-1}\right), & 0 \leq u \leq \delta, \\
\delta \log \left(\delta^{-1}\right)+\bar{\lambda}_{2}^{\prime}(\delta-)(u-\delta), & u>\delta,\end{cases}
\end{gathered}
$$

where $\bar{\lambda}_{2}^{\prime}$ denotes the derivative of function $\bar{\lambda}_{2}$. They are all concave nondecreasing functions satisfying $\int_{0^{+}}\left(1 / \bar{\lambda}_{i}(u)\right) d u=\infty(i=1,2)$. tion.

In the following, we establish the existence and uniqueness theorem of the mild solu-

Theorem 3.3. If (H1)-(H5) hold, then there exists a unique mild solution to (1.1), provided that

$$
K<1 .
$$

Proof. To obtain the existence of the solution to (1.1), we consider the Picard iteration which is defined by

$$
\begin{gathered}
u^{0}(t)=R(t) \varphi(0), \quad t \in[0, T], \\
u^{0}(t)=\varphi(t), \quad t \in[-r, 0],
\end{gathered}
$$

and $u^{n}$ for $n \geq 1$ is defined by

$$
\begin{gathered}
u^{n}(t)=\varphi(t) \quad \text { for } t \in[-r, 0] \\
u^{n}(t)-H\left(t, u_{t}^{n}\right)=R(t)[\varphi(0)-H(0, \varphi)]+\int_{0}^{t} R(t-s) F\left(s, u_{s}^{n-1}\right) d s \\
+\int_{0}^{t} R(t-s) G\left(s, u_{s}^{n-1}\right) d w(s), \quad t \in[0, T] .
\end{gathered}
$$

The proof is divided into the following three steps. 
Step 1. We claim that the sequence $\left\{u^{n}(t), n \geq 0\right\}$ is bounded. From (3.11), for $0 \leq t \leq T$,

$$
\begin{aligned}
E \sup _{0 \leq s \leq t}\left\|u^{n}(s)-H\left(s, u_{s}^{n}(s)\right)\right\|_{\mathbb{H}}^{2} \leq & 3 E \sup _{0 \leq s \leq t}\|R(t)[\varphi(0)-H(0, \varphi)]\|_{\mathbb{H}}^{2} \\
& +3 E \sup _{0 \leq s \leq t}\left\|\int_{0}^{t} R(t-s) F\left(s, u_{s}^{n-1}\right) d s\right\|_{\mathbb{H}}^{2} \\
& +3 E \sup _{0 \leq s \leq t}\left\|\int_{0}^{t} R(t-s) G\left(s, u_{s}^{n-1}\right) d w(s)\right\|_{\mathbb{H}}^{2} \\
= & : 3\left(I_{1}+I_{2}+I_{3}\right) .
\end{aligned}
$$

By (H5),

$$
I_{1} \leq M(1+K)^{2} E\|\varphi\|_{C^{\prime}}^{2}
$$

where $M=\sup _{0 \leq t \leq T}|R(t)|^{2}$.

Note that

$$
E\left(\sup _{0 \leq s \leq t}\left\|u_{s}^{n-1}\right\|_{C}^{2}\right) \leq E\left(\sup _{0 \leq s \leq t}\left\|u^{n-1}(s)\right\|_{\mathbb{H}}^{2}\right)+E\|\varphi\|_{C}^{2} .
$$

By (H3) and the Jensen inequality, we obtain

$$
I_{2} \leq M T \int_{0}^{t} L\left(\tau, E\left(\sup _{0 \leq l \leq \tau}\left\|u^{n-1}(l)\right\|_{\mathbb{H}}^{2}+\|\varphi\|_{C}^{2}\right)\right) d \tau .
$$

By (H3), Liu and Hu [15, Theorem 1.2.5, page 14], and the Jensen inequality, there exists a positive constant $C_{1}$ such that

$$
\begin{aligned}
I_{3} & \leq C_{1} \int_{0}^{t} E G\left(\tau,\left\|u^{n-1}(\tau)\right\|_{\mathbb{H}}^{2}\right) d \tau \\
& \leq C_{1} \int_{0}^{t} E L\left(\tau,\left\|u^{n-1}(\tau)\right\|_{\mathbb{H}}^{2}\right) d \tau \\
& \leq C_{1} \int_{0}^{t} L\left(\tau, E\left\|u^{n-1}(\tau)\right\|_{\mathbb{H}}^{2}\right) d \tau .
\end{aligned}
$$

Since $L$ is continuous monotone nondecreasing in $y$, we obtain

$$
I_{3} \leq C_{1} \int_{0}^{t} L\left(\tau, E\left(\sup _{0 \leq l \leq \tau}\left\|u^{n-1}(l)\right\|_{\mathbb{H}}^{2}+\|\varphi\|_{C}^{2}\right)\right) d \tau \text {. }
$$


Recall that, for $a, b \in X, \varepsilon \in(0,1),\|a-b\|_{X}^{2} \leq 1 /(1-\varepsilon)\|a\|_{X}^{2}+(1 / \varepsilon)\|b\|_{X}^{2}$. Hence, substituting (3.13)-(3.16) into (3.12) yields

$$
\begin{aligned}
& E\left(\sup _{0 \leq s \leq t}\left\|u^{n}(s)\right\|_{\mathbb{H}}^{2}+\|\varphi\|_{C}^{2}\right) \\
& \leq \frac{1}{1-K} E \sup _{0 \leq s \leq t}\left\|u^{n}(s)-H\left(s, u_{s}^{n}\right)\right\|_{\mathbb{H}}^{2}+\frac{1}{K} E \sup _{0 \leq s \leq t}\left\|H\left(s, u_{s}^{n}\right)\right\|_{\mathbb{H}}^{2}+E\|\varphi\|_{C}^{2} \\
& \leq \frac{3 M(1+K)^{2}}{1-K} E\|\varphi\|_{C}^{2}+E\|\varphi\|_{C}^{2}+K E\left(\sup _{0 \leq s \leq t}\left\|u^{n}(s)\right\|_{\mathbb{H}}^{2}+\|\varphi\|_{C}^{2}\right) \\
&+\frac{3\left(M T+C_{1}\right)}{1-K} \int_{0}^{t} L\left(\tau, E\left(\sup _{0 \leq l \leq \tau}\left\|u^{n-1}(l)\right\|_{\mathbb{H}}^{2}+\|\varphi\|_{C}^{2}\right)\right) d \tau \\
& \leq \frac{3 M(1+K)^{2}}{(1-K)^{2}} E\|\varphi\|_{C}^{2}+\frac{1}{1-K} E\|\varphi\|_{C}^{2} \\
&+\frac{3\left(M T+C_{1}\right)}{(1-K)^{2}} \int_{0}^{t} L\left(\tau, E\left(\sup _{0 \leq l \leq \tau}\left\|u^{n-1}(l)\right\|_{\mathbb{H}}^{2}+\|\varphi\|_{C}^{2}\right)\right) d \tau .
\end{aligned}
$$

Assumption (H3-(b)) indicates that there is a solution $x_{t}$ that satisfies

$$
x_{t}=C_{2} E\|\varphi\|_{C}^{2}+C_{3} \int_{0}^{t} L\left(r, x_{r}\right) d r
$$

where $C_{2}=3 M(1+K)^{2} /(1-K)^{2}+1 /(1-K), C_{3}=3\left(M T+C_{1}\right) /(1-K)^{2}$.

Since $E\|\varphi\|_{C}^{2}<\infty$, from (3.17), we have $E\left(\sup _{0 \leq s<t}\left\|u_{s}^{n}\right\|_{C}^{2}\right) \leq x_{t} \leq x_{T}<\infty$, which shows the boundedness of the $\left\{u^{n}(t), n \geq 0\right\}$.

Step 2. We claim that $\left\{u^{n}(t), n \geq 0\right\}$ is a Cauchy sequence. For all $n, m \geq 0$ and $t \in[0, T]$, from (3.11), (H4), and Step 1, we have

$$
\begin{gathered}
E \sup _{0 \leq s \leq t}\left\|u^{n+1}(s)-H\left(s, u_{s}^{n+1}(s)\right)-u^{m+1}(s)+H\left(s, u_{s}^{m+1}(s)\right)\right\|_{\mathbb{H}}^{2} \\
\leq 2 E \sup _{0 \leq s \leq t}\left\|\int_{0}^{s} R(t-s)\left[F\left(s, u_{\tau}^{n+1}\right)-F\left(s, u_{\tau}^{m+1}\right)\right] d \tau\right\|_{\mathbb{H}}^{2} \\
+2 E \sup _{0 \leq s \leq t}\left\|\int_{0}^{t} R(t-s)\left[G\left(\tau, u_{s}^{n}\right)-G\left(\tau, u_{s}^{m}\right)\right] d w(s)\right\|_{\mathbb{H}}^{2} \\
\leq 2\left(M+C_{4}\right) \int_{0}^{s} L\left(\tau, E\left(\sup _{0 \leq l \leq \tau}\left\|u^{n}(l)-u^{m}(l)\right\|_{\mathbb{H}}^{2}\right)\right) d \tau
\end{gathered}
$$


where $C_{4}$ is a generic constant used by Liu and $\mathrm{Hu}[15$, Theorem 1.2.6, page 14]. Therefore,

$$
\begin{aligned}
E & \sup _{0 \leq s \leq t}\left\|u^{2+1}(s)-u^{m+1}(s)\right\|_{\mathbb{H}}^{2} \\
\leq & \frac{1}{1-K} E \sup _{0 \leq s \leq t}\left\|u^{n+1}(s)-H\left(s, u_{s}^{n+1}(s)\right)-u^{m+1}(s)+H\left(s, u_{s}^{m+1}(s)\right)\right\|_{\mathbb{H}}^{2} \\
& +\frac{1}{K} E \sup _{0 \leq s \leq t}\left\|H\left(s, u_{s}^{n+1}\right)-H\left(s, u_{s}^{m+1}\right)\right\|_{\mathbb{H}}^{2} \\
\leq & \frac{2 M\left(1+C_{4}\right)}{1-K} \int_{0}^{s} L\left(\tau, E\left(\sup _{0 \leq l \leq \tau}\left\|u^{n}(l)-u^{m}(l)\right\|_{\mathbb{H}}^{2}\right)\right) d \tau+K E\left(\sup _{0 \leq s \leq t}\left\|u^{n+1}(s)-u^{m+1}(s)\right\|_{\mathbb{H}}^{2}\right) .
\end{aligned}
$$

Let

$$
z(t)=\lim _{n, m \rightarrow+\infty} \sup \left(\sup _{0 \leq s \leq t}\left\|u^{n}(s)-u^{m}(s)\right\|_{\mathbb{H}}^{2}\right) .
$$

By assumption (H4-(b)) and the Fatou lemma, we have

$$
z(t) \leq C_{5} \int_{0}^{t} \lambda(s, z(s)) d s
$$

where $C_{5}=2 M\left(1+C_{4}\right) /(1-K)^{2}$. By assumption (H4-(b)) we obtain $z(t)=0$. This shows that $\left\{u^{n}(t), n \geq 0\right\}$ is Cauchy.

Step 3. We claim the existence and uniqueness of the solution to (1.1). The Borel-Cantelli lemma shows that, as $n \rightarrow \infty, u^{n}(t) \rightarrow u(t)$ holds uniformly for $0 \leq t \leq T$. Hence, taking limits on both sides of (3.11), we obtain that $u(t)$ is a solution to (1.1). This shows the existence. The uniqueness of the solution could be obtained by the same procedure as Step 2 . The proof is complete.

Remark 3.4. If $\lambda(t, u)=K_{1} u$ for some constant $K_{1}$, then condition (H4) implies a global Lipschitz condition.

\section{Application}

We conclude this work with an example of the form

$$
\begin{gathered}
\frac{\partial}{\partial t}\left[x(t, \xi)-\int_{-r}^{0} h(t, x(t+\theta, \xi)) d \theta\right]=\frac{\partial^{2}}{\partial \xi^{2}}\left[x(t, \xi)-\int_{-r}^{0} h(t, x(t+\theta, \xi)) d \theta\right] \\
+\int_{0}^{t} b(t-s) \frac{\partial^{2}}{\partial \xi^{2}}\left[x(s, \xi)-\int_{-r}^{0} h(t, x(t+\theta, \xi)) d \theta\right] d s
\end{gathered}
$$




$$
\begin{aligned}
& +\int_{-r}^{0} f(t, x(t+\theta, \xi)) d \theta+g(t, x(t+\theta, \xi)) d w(t), \quad \text { for } t \geq 0, \xi \in[0, \pi], \\
& x(t, 0)-\int_{-r}^{0} h(t, x(t+\theta, 0)) d \theta=0, \quad \text { for } t \geq 0, \\
& x(t, \pi)-\int_{-r}^{0} h(t, x(t+\theta, \pi)) d \theta=0, \quad \text { for } t \geq 0, \\
& x(\theta, \xi)=x_{0}(\theta, \xi), \quad \text { for } \theta \in[-r, 0], \xi \in[0, \pi],
\end{aligned}
$$

where $w(t)$ denotes an $\mathbb{R}$-valued Brownian motion, $h, f, g: \mathbb{R}^{+} \times \mathbb{R} \rightarrow \mathbb{R}$ are continuous functions, $b: \mathbb{R}^{+} \rightarrow \mathbb{R}$ is continuous, and $x_{0}:[-r, 0] \times[0, \pi] \rightarrow \mathbb{R}$ is a given continuous function such that $x_{0}(\cdot) \in L^{2}([0, \pi])$ is $\mathcal{F}_{0}$-measurable and satisfies $E\left\|x_{0}\right\|^{2}<\infty$.

Let $\mathbb{H}=L^{2}([0, \pi])$ with the norm $\|\cdot\|$ and $e_{n}:=\sqrt{2 / \pi} \sin (n x),(n=1,2,3, \ldots)$ denote the completed orthonormal basis in $\mathbb{H}$. Let $w(t):=\sum_{n=1}^{\infty} \sqrt{\lambda_{n}} \beta_{n}(t) e_{n}\left(\lambda_{n}>0\right)$, where $\beta_{n}(t)$ are one-dimensional standard Brownian motion mutually independent on a usual complete probability space $\left(\Omega, \mathcal{F},\left\{\mathcal{F}_{t}\right\}_{t \geq 0}, \mathbb{P}\right)$.

Define $A: \mathbb{H} \rightarrow \mathbb{H}$ by $A=\partial^{2} / \partial z^{2}$, with domain $D(A)=H^{2}([0, \pi]) \cap H_{0}^{1}([0, \pi])$.

Then $A h=-\sum_{n=1}^{\infty} n^{2}\left\langle h, e_{n}\right\rangle e_{n}, h \in D(A)$, where $e_{n}, n=1,2,3, \ldots$, is also the orthonormal set of eigenvectors of $A$. It is wellknown that $A$ is the infinitesimal generator of a strongly continuous semigroup on $\mathbb{H}$; thus, $(H 1)$ is true.

Let $B: D(A) \subset \mathbb{H} \rightarrow \mathbb{H}$ be the operator defined by $B(t)(z)=b(t) A z$ for $t \geq 0$ and $z \in D(A)$.

We suppose that

(i) there exists a positive constant $L_{h}, 0<\sqrt{\pi} L_{h} r<1$, such that

$$
\left|h\left(t, \zeta_{1}\right)-h\left(t, \zeta_{2}\right)\right| \leq L_{h}\left|\zeta_{1}-\zeta_{2}\right|, \quad \text { for } t \geq 0, \zeta_{1}, \zeta_{2} \in \mathbb{R}
$$

(ii) there exists a constant $L_{f}, 0<\sqrt{\pi} L_{f} r<1$, such that

$$
\left|f\left(t, \zeta_{1}\right)-f\left(t, \zeta_{2}\right)\right|^{2} \leq L_{f} \lambda\left(t,\left\|\zeta_{1}-\zeta_{2}\right\|_{C}^{2}\right), \quad \text { for } t \geq 0, \zeta_{1}, \zeta_{2} \in \mathbb{R}
$$

(iii) for $t \geq 0$ and $\zeta_{1}, \zeta_{2} \in \mathbb{R},\left|g\left(t, \zeta_{1}\right)-g\left(t, \zeta_{2}\right)\right|^{2} \leq \lambda\left(t,\left\|\zeta_{1}-\zeta_{2}\right\|_{C}^{2}\right)$.

Let $C=C([-r, 0] ; \mathbb{H})$ and define the operators $H, F, G: \mathbb{R}^{+} \times C \rightarrow \mathbb{H}$ by

$$
\begin{gathered}
H(t, \phi)(\xi)=\int_{-r}^{0} h(t, \phi(\theta)(\xi)) d \theta, \quad \text { for } \xi \in[0, \pi], \phi \in C, \\
F(t, \phi)(\xi)=\int_{-r}^{0} f(t, \phi(\theta)(\xi)) d \theta, \quad \text { for } \xi \in[0, \pi], \phi \in C, \\
G(t, \phi)(\xi)=g(t, \phi(\theta)(\xi)) .
\end{gathered}
$$


If we put

$$
\begin{array}{cl}
u(t)=x(t, \xi), & \text { for } t \geq 0, \xi \in[0, \pi], \\
\varphi(\theta)(\xi)=x_{0}(\theta, \xi), & \text { for } \theta \in[-r, 0], \xi \in[0, \pi] .
\end{array}
$$

then (4.1) takes the following abstract form:

$$
\begin{aligned}
& d\left[u(t)-H\left(t, u_{t}\right)\right] \\
& =A\left[u(t)-H\left(t, u_{t}\right)\right] d t+\left[\int_{0}^{t} B(t-s)\left[u(s)-H\left(s, u_{s}\right)\right] d s+F\left(t, u_{t}\right)\right] d t \\
& +G\left(t, u_{t}\right) d w(t), \quad \text { for } t \in[0, T] \\
& u_{0}=\varphi .
\end{aligned}
$$

Moreover, if $b$ is bounded and $C^{1}$ function such that $b^{\prime}$ is bounded and uniformly continuous, then (H1) and (H2) are satisfied, and hence, by Theorem 2.2, (2.3) has a resolvent operator $(R(t))_{t \geq 0}$ on $\mathbb{H}$. Thus, all the assumptions of Theorem 3.3 are fulfilled. Therefore, the existence of a unique mild solution of (4.1) follows.

\section{References}

[1] Y. Ren and L. Chen, "A note on the neutral stochastic functional differential equation with infinite delay and Poisson jumps in an abstract space," Journal of Mathematical Physics, vol. 50, Article ID 082704, 2009.

[2] Y. Ren and N. Xia, "Existence, uniqueness and stability of the solutions to neutral stochastic functional differential equations with infinite delay," Applied Mathematics and Computation, vol. 210, no. 1, pp. 72 79, 2009.

[3] L. Hu and Y. Ren, "Existence results for impulsive neutral stochastic functional integro-differential equations with infinite delays," Acta Applicandae Mathematicae, vol. 111, no. 3, pp. 303-317, 2010.

[4] A. Lin, Y. Ren, and N. Xia, "On neutral impulsive stochastic integro-differential equations with infinite delays via fractional operators," Mathematical and Computer Modelling, vol. 51, no. 5-6, pp. 413-424, 2010.

[5] H. Bao and J. Cao, "Existence and uniqueness of solutions to neutral stochastic functional differential equations with infinite delay," Applied Mathematics and Computation, vol. 215, no. 5, pp. 1732-1743, 2009.

[6] J. Bao and Z. Hou, "Existence of mild solutions to stochastic neutral partial functional differential equations with non-Lipschitz coefficients," Computers $\mathcal{E}$ Mathematics with Applications, vol. 59, no. 1, pp. 207-214, 2010.

[7] T. Taniguchi, "Successive approximations to solutions of stochastic differential equations," Journal of Differential Equations, vol. 96, no. 1, pp. 152-169, 1992.

[8] J. Turo, "Successive approximations of solutions to stochastic functional-differential equations," Periodica Mathematica Hungarica, vol. 30, no. 1, pp. 87-96, 1995.

[9] G. Cao, K. He, and X. Zhang, "Successive approximations of infinite dimensional SDEs with jump," Stochastics and Dynamics, vol. 5, no. 4, pp. 609-619, 2005.

[10] F. Jiang and Y. Shen, "A note on the existence and uniqueness of mild solutions to neutral stochastic partial functional differential equations with non-Lipschitz coefficients," Computers $\mathcal{E}$ Mathematics with Applications, vol. 61, no. 6, pp. 1590-1594, 2011.

[11] T. E. Govindan, "Almost sure exponential stability for stochastic neutral partial functional differential equations," Stochastics, vol. 77, no. 2, pp. 139-154, 2005. 
[12] J. Bao and Z. Hou, "Existence of mild solutions to stochastic neutral partial functional differential equations with non-Lipschitz coefficients," Computers $\mathcal{E}$ Mathematics with Applications, vol. 59, no. 1, pp. 207-214, 2010.

[13] R. C. Grimmer, "Resolvent operators for integral equations in a Banach space," Transactions of the American Mathematical Society, vol. 273, no. 1, pp. 333-349, 1982.

[14] R. C. Grimmer and A. J. Pritchard, "Analytic resolvent operators for integral equations in Banach space," Journal of Differential Equations, vol. 50, no. 2, pp. 234-259, 1983.

[15] A. Lin and L. Hu, "Existence results for impulsive neutral stochastic functional integro-differential inclusions with nonlocal initial conditions," Computers \& Mathematics with Applications, vol. 59, no. 1, pp. 64-73, 2010. 


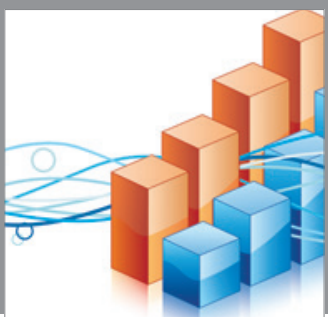

Advances in

Operations Research

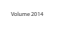

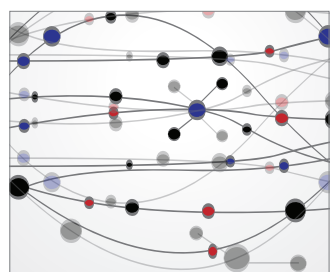

\section{The Scientific} World Journal
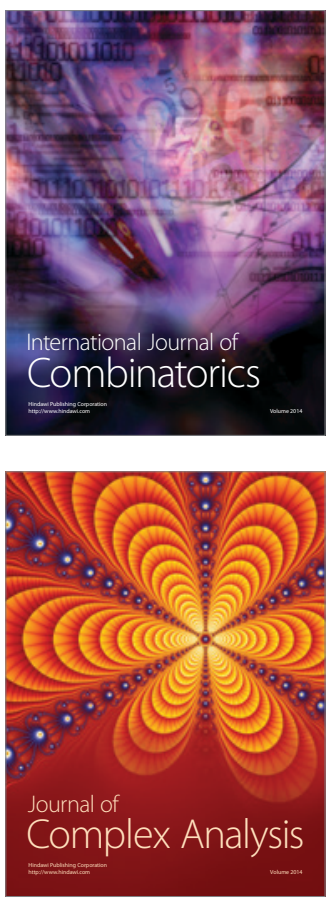

International Journal of

Mathematics and

Mathematical

Sciences
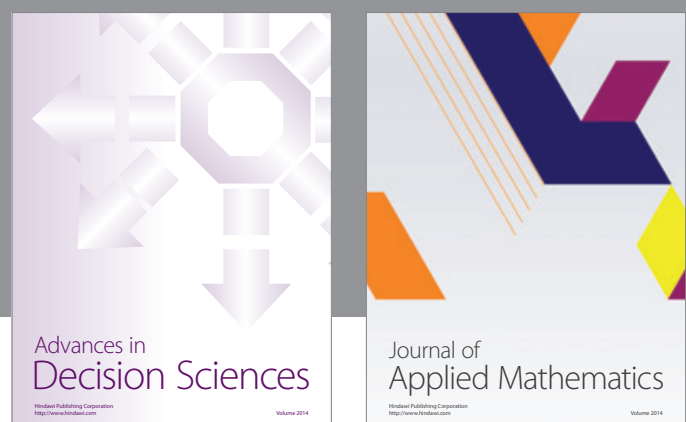

Journal of

Applied Mathematics
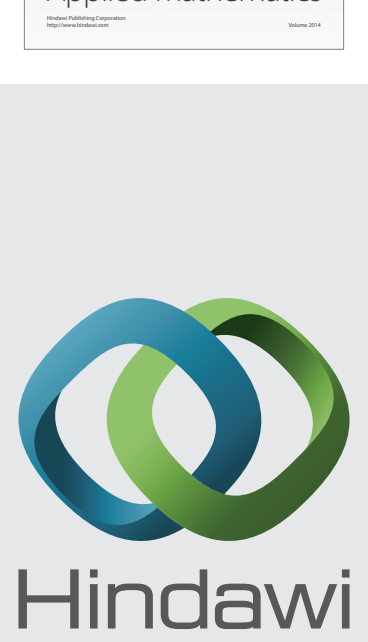

Submit your manuscripts at http://www.hindawi.com
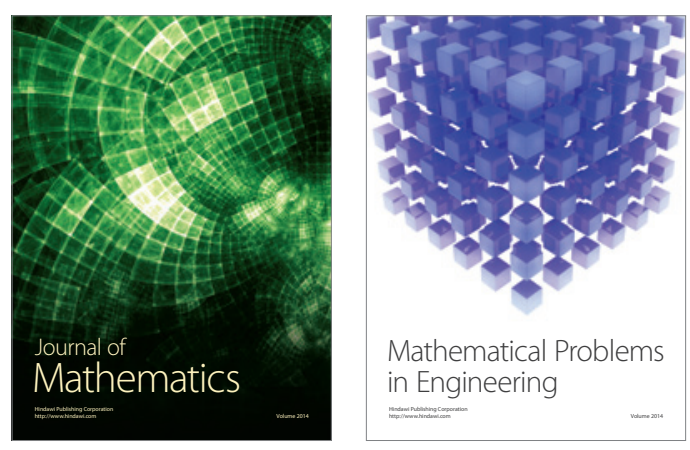

Mathematical Problems in Engineering
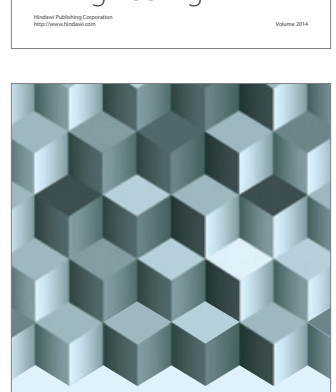

Journal of

Function Spaces
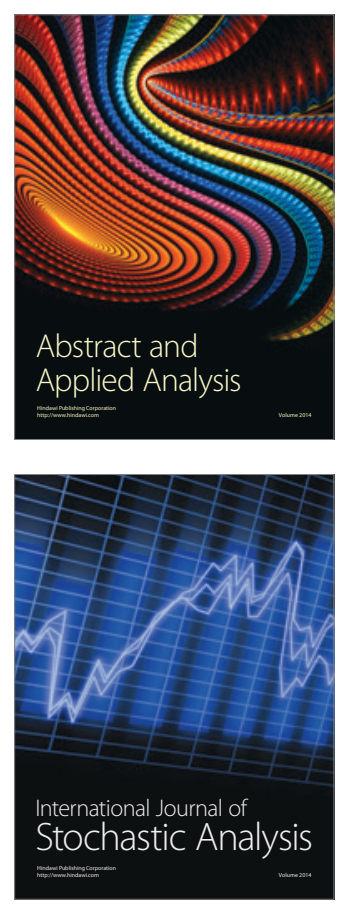

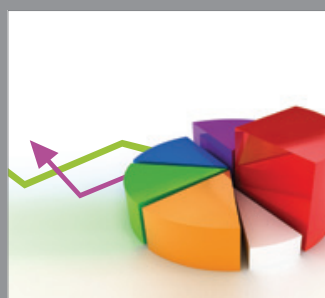

ournal of

Probability and Statistics

Promensencen
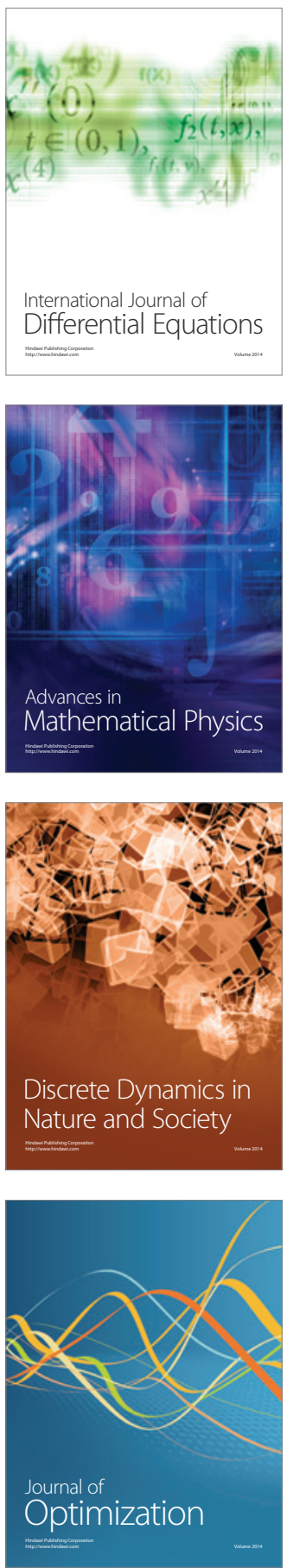